\title{
Cuando la contigüidad no es suficiente: bloqueo en relaciones de equivalencia*
}

When Contiguity is not Enough: Blocking Equivalence Relations

Recibido: mayo 15 de 2012 | Revisado: diciembre 10 de 2012 | Aceptado: enero 17 de 2012

\author{
Diana Marcela Delgado Delgado ** \\ IVÁN FELIPE MEDINA ARbOlEDA *** \\ Fundación Universitaria Konrad Lorenz, Bogotá, Colombia
}

doi:10.11144/Javeriana.UPSY12-2.ccns

Para citar este artículo: Delgado, D. M. \& Medina, I. F. (2013). Cuando la contigüidad no es suficiente: bloqueo en relaciones de equivalencia. Universitas Psychologica, 12(2), 613-626.

* Reconocimientos: Este artículo corresponde a la investigación institucional "Evaluación del efecto de bloqueo durante la adquisición de relaciones de equivalencia", auspiciada en su totalidad por la Fundación Universitaria Konrad Lorenz (Fondo: 95101101).

*** Fundación Universitaria Konrad Lorenz, Colombia. Investigadora, Laboratorio de Comportamiento Humano. Carrera 9 Bis No. 62 -43. Bogotá, Colombia.E-mail: diana.delgado@konradlorenz.edu.co

**** Profesor Asistente. Fundación Universitaria Konrad Lorenz.E-mail: ivanf.medinaa@konradlorenz. edu.co

\section{RESUMEN}

El fenómeno de bloqueo describe una circunstancia en la cual se impide o se dificulta el la transferencia de funciones entre eventos que se han presentado en una relación de contigüidad espacio-temporal. Desde una perspectiva teórica y empírica, el efecto bloqueo no debería predecirse en la adquisición de clases de estímulos aun cuando algunos de los estímulos de la misma clase se presenten simultáneamente. El presente estudio examina si la historia adicional de entrenamiento A-EI bien sea previa o posterior al entrenamiento con un estímulo muestra compuesto AX, es la variable crítica en la presentación del efecto bloqueo. Los desempeños obtenidos en las pruebas de bloqueo por los participantes de un grupo expuesto al procedimiento de bloqueo, un grupo expuesto al procedimiento de bloqueo invertido, y un grupo control, sugieren que el entrenamiento adicional con un solo elemento del compuesto no impide la inclusión de todos los estímulos dentro de la clase. Los casos en los que se observó el efecto bloqueo se interpretan en términos de tipos de percepciones elementales y configurales.

\section{Palabras clave autores}

Efecto bloqueo, equivalencia, transferencia de funciones, tipo-respondiente,

estímulos compuestos, relaciones derivadas, igualación a la muestra, respuestas elementales, respuestas configurales.

Palabras clave descriptores

Condicionamiento clásico, teoría del aprendizaje, igualación a la muestra, atención visual.

\section{A B S T R AC T}

The blocking effect describes a circumstance which impedes function transfer among events that are part of a relation of spatiotemporal contiguity. However, theoretical and empirical evidence in stimulus class acquisition predicts that the blocking effect should not be observed even if some of the stimuli in the class are presented simultaneously as compound stimuli. The present study examines if additional A-EI training, whether prior or following training with a compound sample AX, constitutes a critical variable in the occurrence of the blocking effect. Performances in blocking tests by a group exposed to the blocking procedure, a group exposed to the backward blocking procedure and a control group were compared. Findings suggest that additional training with one member of the compound does not impede responding to all stimuli as members of the class. Instances where the blocking effect was observed are interpreted in terms of configural and elemental types of responding.

Key words authors

Blocking Effect, Equivalence, Function Transfer, Respondent-Type, Compound Stimuli, Derived Relations, Matching To Sample, Elemental Responding, Configural Responding.

Key words plus

Classical Conditioning, Learning Theory, Matching To Sample, Visual Attention. 
El efecto bloqueo ocurre cuando no se observan respuestas condicionadas ante uno de los estímulos (X) que hace parte de un compuesto (AX), dada una historia previa de condicionamiento con el otro elemento del compuesto (A). Las primeras explicaciones de este hallazgo hacen referencia a la redundancia del estímulo X para predecir el estimulo incondicionado (EI) durante el entrenamiento AX-EI. Esto sucede debido a que durante la exposición previa a la asociación A-EI, el estímulo A adquiere una función predictora con respecto a la ocurrencia del EI y por lo tanto su presentación es suficiente para la elicitación de respuestas con respecto del EI (Kamin, 1968, 1969).

El efecto bloqueo contradice uno de los supuestos del condicionamiento clásico al poner en evidencia que la contigüidad espacio-temporal no es una condición suficiente para el mismo. Por esta razón, se han propuesto varios modelos explicativos que dan cuenta de este fenómeno en términos de perspectivas más actuales del condicionamiento clásico (p. ej., Blaisdell, Gunther \& Miller, 1999; Mackintosh, 1975; McLaren \& Mackintosh, 2000; Rescorla, 1999; Stout \& Miller, 2007). Adicionalmente, variaciones de tipo procedimental relacionadas con el orden temporal y espacial de presentación de los estímulos, han permitido examinar las circunstancias bajo las cuales se dificulta el aprendizaje o la transferencia de funciones estimulativas tanto en animales (Pineño, Urushihara, Stout, Fuss \& Miller, 2006) como en humanos (Livesey \& Boakes, 2004; Rehfeldt, Dixon, Hayes \& Steele, 1998; Singh \& Solman, 1990).

Desde las perspectivas tradicionales del condicionamiento clásico, el condicionamiento de la respuesta electrodérmica se ha utilizado ampliamente como modelo para examinar el efecto de bloqueo en humanos (Davey \& Arulampalam, 1982; Lachnit \& Lober, 2001). Las aproximaciones contemporáneas, por otra parte, utilizan el procedimiento de bloqueo (entre otros procedimientos de condicionamiento pavloviano) para examinar cómo contribuye la transferencia de funciones a la comprensión de fenómenos relacionados con la cognición humana. En estos estudios, el EI no constituye un estímulo biológicamente relevante, ni evoca necesariamente una respuesta refleja (Livesey \& Boakes, 2004; Rehfeldt et al., 1998; Rescorla, 1988; Shanks, 1985; Singh \& Solman, 1990; Wasserman \& Berglan, 1998).

Por ejemplo, en un estudio realizado por $\mathrm{Ar}$ cediano, Matute y Miller (1997), los participantes presionaban una letra en el teclado del computador para disparar un arma láser en un juego de video, pero debían dejar de disparar ante la presentación de un estímulo visual. Consistentemente con las predicciones del efecto bloqueo, se observó menor supresión de la respuesta ante un estímulo auditivo, después de la presentación conjunta de los estímulos auditivo y visual (ver también, Crookes \& Moran, 2003; Kruschke, Kapperman \& Hetrick, 2005; Wilson \& Alexander, 2008). Sin embargo, dado que no en todos los estudios con humanos se observa el efecto de bloqueo para todos los participantes, no ha sido posible establecer con claridad cuáles son las circunstancias que impiden o facilitan el aprendizaje en humanos bajo estas condiciones de exposición a los estímulos (Laane, Aru \& Dickinson, 2010; Rehfeldt et al., 1998).

Estudios posteriores han evaluado el impacto del efecto bloqueo sobre el aprendizaje de relaciones entre estímulos verbales. Por ejemplo, Singh y Solman (1990), evaluaron el efecto bloqueo en el aprendizaje de la lectura, en niños con déficit en el desarrollo. Específicamente, los autores examinaron si la historia previa de asociaciones entre una imagen y una palabra hablada impedía a los niños responder en términos de la relación palabra escritapalabra hablada, después de un entrenamiento en el que se presentaba conjuntamente la imagen y la palabra escrita, seguidas de la palabra hablada. Un examen de los efectos diferenciales de la presentación demorada o simultánea de la imagen y la palabra escrita (i. e., los elementos del estímulo compuesto) indicó que los niños expuestos a condiciones de menor demora entre la presentación de imágenes y palabras habladas, respondieron con menor precisión en términos de la relación palabra escrita-palabra hablada. En contraste, se observó un mayor número de vocalizaciones adecuadas al incrementar el intervalo interestímulo entre la imagen y la palabra durante el entrenamiento (ver también, Didden, Prinsen \& Sigafoos, 2000). 
Hallazgos similares se han reportado en estudios en los que se examina el efecto bloqueo en la atribución de juicios causales. En estos estudios, se examina si los participantes atribuyen causalidad a cada uno de los elementos de un evento compuesto, el cual se ha presentado seguido de una consecuencia o resultado (De Houwer, Beckers \& Glautier, 2002; Glautier, 2002; Livesey \& Boakes, 2004). Para este propósito se utiliza el procedimiento estándar del efecto bloqueo. En los ensayos de la fase de preentrenamiento, los participantes observan que un evento $\mathrm{X}$ es seguido de/produce, una consecuencia. Durante la fase de entrenamiento, los ensayos incluyen la presentación conjunta del evento $\mathrm{X}$ con un evento $\mathrm{Y}$ adicional, seguidos de la misma consecuencia. En la prueba de bloqueo se evalúa la atribución de causalidad a X y a Y de manera independiente, dada la ocurrencia de la consecuencia.

Si bien el efecto bloqueo no se ha observado de manera consistente en humanos, algunos autores sugieren que responder a los estímulos de la fase de entrenamiento, bien sea como a eventos diferenciables entre sí o como a partes de un solo estímulo compuesto, puede constituir un aspecto determinante en el bloqueo de la respuesta condicionada. Sin embargo, los hallazgos y sus interpretaciones continúan siendo fuente de controversia.

Singh y Solman (1990), por ejemplo, muestran que el elemento crítico en la observación del fenómeno es la presentación conjunta y simultánea de los estímulos que hacen parte del evento compuesto durante la fase de entrenamiento. Sin embargo, los estudios de De Houwer et al. (2002) y de Livesey y Boakes (2004) muestran que es más probable que se observe el efecto bloqueo cuando los participantes responden a los estímulos del compuesto de manera independiente.

Las relaciones entre estímulos arbitrarios implicadas en el lenguaje no referencial y la transferencia de este aprendizaje a estímulos nuevos y a relaciones no entrenadas, se han abordado través de los estudios de relaciones de equivalencia, utilizando procedimientos de igualación a la muestra (ver, Cepeda-Islas et al., 2009; Escuer-Acín, GarcíaGarcía, Bohórquez-Zayas \& Gutiérrez-Domínguez,
2006; García \& Benjumea, 2007; Sidman, 1992, 2000; Sidman \& Tailby, 1982; Valero-Aguayo \& Luciano-Soriano, 1992; Vives-Montero, ValeroAguayo \& Ascanio, 2011; Wulfert \& Hayes, 1988). Los estudios realizados utilizando procedimientos pavlovianos de igualación a la muestra han mostrado sistemáticamente que la emergencia de relaciones no entrenadas entre estímulos arbitrarios puede explicarse de manera suficiente por procesos de condicionamiento clásico (Delgado \& Medina, 2011; Delgado, Medina \& Soto, 2011; Leader \& Barnes-Holmes, 2001; Tonneau, Arreola \& Martínez, 2006; Tonneau \& González, 2004).

Más aún, podría decirse que el procedimiento de bloqueo no es significativamente diferente a algunos entrenamientos de adquisición de clases de estímulos. Dado un entrenamiento inicial en la relación A-C, seguido de un entrenamiento en AB-C (reemplazando al EI por C), el efecto bloqueo prediría que la relación B-C no sería adquirida. Procedimentalmente, dos tipos de arreglos experimentales de igualación a la muestra pueden ilustrar la fase de entrenamiento $\mathrm{AB}-\mathrm{C}$ del procedimiento de bloqueo: a) los procedimientos con estímulos compuestos, con $\mathrm{AB}$ como estímulo muestra compuesto y b) procedimientos de control contextual en donde A y B podrían corresponder a estímulos contextuales y condicionales respectivamente, en una contingencia operante de cinco términos.

Rehfeldt et al. (1998) evaluaron el efecto de bloqueo en la formación de relaciones derivadas, utilizando muestras compuestas en los ensayos de discriminaciones condicionadas. Para este propósito, entrenaron las relaciones A-B, AX-B y B-C, empleando el procedimiento tradicional de igualación a la muestra. Para evaluar el efecto bloqueo se presentó el estímulo X de manera adyacente a la muestra A, para formar el compuesto AX. Las pruebas de bloqueo incluyeron relaciones de simetría, transitividad y equivalencia entre los estímulos A-B-C y entre los estímulos X-B-C.

Los resultados en las pruebas de relaciones derivadas mostraron un efecto de bloqueo solamente para la mitad de los participantes. Posiblemente, debido a que la posición (izquierda/derecha) de los estímulos A y X de la muestra compuesta se cambió 
de manera aleatoria en cada ensayo, algunos participantes respondieron a cada uno de los elementos de la muestra compuesta separadamente, lo cual facilitó la inclusión de ambos elementos del compuesto en las clases de equivalencia. No es claro, sin embargo, cuáles son las variables que contribuyeron a que los participantes respondieran a AX como un solo estímulo, o a cada uno de sus elementos de manera separada.

Un hallazgo interesante es que los participantes en los que se observó el efecto bloqueo también obtuvieron un desempeño por debajo del criterio en las pruebas de relaciones simétricas entre elementos del compuesto, A-X y X-A (Rehfeldt et al., 1998). Estos hallazgos son consistentes con los estudios que muestran que el efecto bloqueo se observa con mayor probabilidad cuando los estímulos del compuesto se presentan de manera simultánea. En términos teóricos, por otra parte, los hallazgos de este estudio aportan evidencia adicional a favor del involucramiento de procesos de condicionamiento respondiente en la adquisición de clases de equivalencia (Delgado \& Medina, 2011; Delgado et al., 2011; Tonneau \& González, 2004; Tonneau et al., 2006).

En la mayoría de los estudios de equivalencia con muestras compuestas, los participantes responden separadamente a cada elemento del compuesto como a un miembro de la clase de equivalencia (Augustson, Dougher \& Markham, 2000; Carpentier, Smeets \& Barnes-Holmes, 2005; Dougher \& Markham, 1994; Hernández, Céspedes \& Prieto, 2007; Markham \& Dougher, 1993; Markham, Dougher \& Augustson, 2002; Stromer \& Stromer, 1990). Sin embargo, se debe tener en cuenta que solamente en el estudio de Rehfeldt et al. (1998), se entrenó previamente la relación entre un solo elemento del compuesto y otro estímulo de la misma clase (i. e., A-B, y posteriormente AX-B). Esto permite la evaluación de la historia previa con un solo elemento del compuesto como un factor importante en la explicación del efecto bloqueo.

La predicción del modelo de bloqueo en relaciones de equivalencia no cuenta con apoyo empírico suficiente en la investigación con humanos. A la luz de las teorías de relaciones de equivalencia y otras relaciones derivadas, no es claro cómo se puede dar sustento lógico y conceptual a las predicciones del efecto de bloqueo. Las condiciones procedimentales que predicen el fenómeno de bloqueo en humanos tampoco son claramente identificables. De acuerdo con las investigaciones realizadas hasta el momento, dos aspectos parecen ser influyentes en la presentación del efecto bloqueo: a) la historia previa de aprendizaje con uno de los elementos del compuesto y/o b) el responder a los estímulos perceptualmente de manera configural o elemental, esto es, responder a los elementos del compuesto, ya sea como a un solo evento o como a estímulos independientes (De Houwer et al., 2002; Livesey \& Boakes, 2004; Melchers, Shanks \& Lachnit, 2008).

El presente estudio tiene como propósito identificar las condiciones que predicen el bloqueo en el proceso de transferencia de funciones y, específicamente, en la adquisición de clases de equivalencia. Si el efecto bloqueo ocurre sistemáticamente en humanos, este permitiría predecir las condiciones bajo las cuales se impide la transferencia de funciones en la formación de clases funcionales de estímulos arbitrarios. Fundamentalmente, se examinará la hipótesis de la redundancia de $\mathrm{X}$ en la predicción del EI, es decir el primero de los aspectos mencionados arriba, con una dimensión adicional.

Se evaluará si este efecto de redundancia se produce únicamente debido a una historia previa de asociaciones entre uno de los elementos del compuesto y otro estímulo, o si también ocurre cuando el entrenamiento con el estímulo compuesto ocurre primero, seguido de la exposición a asociaciones entre un solo elemento del compuesto y el otro estímulo. Este último arreglo experimental corresponde al procedimiento de bloqueo invertido, en el cual se cambia el orden de presentación de las fases de preentrenamiento (A-EI) y entrenamiento (AX-EI), es decir, se presenta primero AX-EI y luego A-EI.

Si bien algunos estudios sugieren que al utilizar el procedimiento inverso de bloqueo no se observa el efecto en animales pero sí en humanos (Denniston, Miller \& Matute, 1996), los estudios con humanos que comparan los efectos de estos dos 
procedimientos en la transferencia de funciones son escasos. El presente estudio examina si el efecto bloqueo en la formación de clases de equivalencia se observa como resultado de una historia previa y/o de la exposición posterior a asociaciones entre un elemento del compuesto y un miembro de la clase, dado un entrenamiento (previo o posterior al anterior) en el cual el compuesto AX se presenta en conjunto con un miembro de la clase.

Para esto se utilizará la estructura general del procedimiento utilizado por Rehfeldt et al. (1998) descrito previamente. En este procedimiento, se entrena a los participantes en las relaciones A-B, AX-B y B-C y se evalúan las relaciones de simetría, transitividad y equivalencia entre A-B-C y X-B-C. Un mayor número de respuestas correctas en las pruebas con los estímulos A-B-C que con los X$\mathrm{B}-\mathrm{C}$ se considera como indicativo de bloqueo en la transferencia de funciones entre X y los demás miembros de la clase.

Se consideró necesario introducir algunas modificaciones al procedimiento general de Rehfeldt et al. (1998) para dar respuesta a los propósitos de este estudio. (a) A diferencia del estudio de Rehfeldt et al. (1998), los estímulos A y X de la muestra AX no cambian de posición (izquierda/derecha) en cada ensayo. Esto, con el fin de reducir la posibilidad de que los participantes respondan a los estímulos de manera independiente. (b) Dado que se asume la transferencia de funciones como elemento explicativo suficiente de la adquisición de relaciones entrenadas y no entrenadas entre estímulos, se utiliza el procedimiento de igualación a la muestra respondiente (Delgado \& Medina, 2011; Delgado et al., 2011; Tonneau \& González, 2004). (c) Se manipula el orden de los entrenamientos en dos grupos para examinar si existen efectos diferenciales de los procedimientos de bloqueo y bloqueo invertido, sobre el bloqueo en la formación de relaciones derivadas. Por último, (d) el desempeño de los participantes en el grupo de bloqueo y de bloqueo invertido se compara con el de un grupo control. En el procedimiento del grupo control se entrenan únicamente las relaciones AX-B y B-C sin bloques de ensayos adicionales para la relación A-B.

\section{Método}

\section{Participantes}

Un total de 24 estudiantes de pregrado de un establecimiento de Educación Superior participaron voluntariamente en el estudio a cambio de una bonificación en una de sus asignaturas. Se tuvieron en cuenta dos criterios de exclusión:

1. Experiencia previa experimental o conocimiento de las características del método de igualación a la muestra.

2. El segundo criterio está relacionado con el desempeño de los estudiantes en la tarea experimental. Después de cada bloque de ensayos de entrenamiento se presentaron cuatro ensayos de evaluación de la relación anteriormente entrenada, los cuales sirvieron como un filtro para continuar con la tarea experimental. Se consideró necesario incluir este filtro dado que si los participantes no atienden a los estímulos presentados o no realizan las actividades implicadas en la tarea, las pruebas no aportan información sobre las variables relevantes para la investigación.

\section{Instrumentos}

Las tareas experimentales se programaron con el software LabView versión 2011. Se utilizaron ocho estímulos arbitrarios previamente asignados por los experimentadores a dos clases de equivalencia de tres miembros cada una (A1-B1-C1; A2-B2-C2). Los estímulos X1 y X2 se presentaron a la derecha de los estímulos A1 y A2 para formar los estímulos muestra compuestos AX1 y AX2, presentados durante la fase de entrenamiento.

Cada estímulo es una figura compuesta por líneas negras entrecruzadas, la cual se presentó dentro de un recuadro de fondo blanco de $4 \times 4 \mathrm{~cm}$. En todos los ensayos los estímulos se presentaron sobre un fondo de pantalla de color gris. Las figuras utilizadas como estímulos fueron tomadas de estudios previos realizados por los autores (ver, Delgado \& Medina, 2011; Delgado et al., 2011). En la Figura 1 se presentan los ocho estímulos designados alfa- 
numéricamente para facilitar su referenciación en el resto del manuscrito.

\section{Procedimiento}

Se ubicó a cada participante frente a un computador. Cada computador se encontraba aislado de los demás por divisiones modulares. De esta manera, fue posible que varios participantes desarrollaran la tarea experimental simultáneamente sin interferencias o distracciones auditivas o visuales adicionales a los estímulos de la tarea.

Antes de comenzar la tarea experimental un auxiliar de investigación informó a cada participante que el objetivo de la investigación era examinar las circunstancias involucradas en el aprendizaje de relaciones entre eventos. Adicionalmente, se indicó que una vez comenzada la tarea, esta debía concluirse sin interrupciones, pausas o preguntas. En cualquier momento los participantes podían abandonar la tarea y cerrar el aplicativo.

Después de leer y firmar el consentimiento informado cada participante recibió la siguiente instrucción:

A continuación observará algunas figuras en la parte superior-central del monitor y otras dos figuras diferentes en la parte inferior. Usted notará que la(s) figura(s) de la parte superior está(n) relacionadas de alguna manera con una de las dos figuras que aparecen debajo. Observe con atención estas relaciones. Cuando el programa se lo indique usted debe seleccionar con el mouse del computador una de las dos figuras de la parte inferior con base en sus observaciones anteriores. Ahora puede comenzar.

Los participantes se dividieron aleatoriamente en tres grupos de ocho personas cada uno: el grupo control, el grupo bloqueo y el grupo bloqueo invertido. La tarea experimental para cada grupo varió en algunas de las relaciones entrenadas y en su orden de presentación.

Para los tres grupos, BL (Bloqueo), BL-I (Bloqueo Invertido y CL (Control), la tarea experimental consistió en bloques de ensayos de entrenamiento seguidos por bloques de pruebas. Para los ensayos de entrenamiento se utilizó el procedimiento de igualación a la muestra respondiente. Este procedimiento consiste en la presentación de un estímulo muestra en la parte central superior de la pantalla (p. ej., A1) seguido dos segundos después, por la presentación de dos estímulos de comparación en la parte inferior (p. ej., B1 y B2). Uno de estos dos estímulos hace parte de la misma clase de equivalencia con estímulo muestra (p. ej., A1-B1). Un recuadro rojo alrededor del estímulo muestra y el estímulo de comparación de la misma clase aparece durante dos segundos. Después de un intervalo interensayo de dos segundos, se presenta un nuevo ensayo de entrenamiento. La posición izquierda o derecha de los estímulos de comparación se aleatorizó entre ensayos. Se presentaron 10 ensayos para entrenar cada una de las relaciones A-B, AX-B y B-C. Para cada bloque de 10 ensayos cada uno de los estímulos se presentó como muestra cinco veces de manera semialeatoria. A continuación, se describen las variaciones en el arreglo experimental para cada grupo.

Grupo BL. Después de entrenar las relaciones A-B se presentaron cuatro ensayos de prueba A-B. En esta prueba filtro el participante debía seleccionar con el mouse el estímulo de comparación que hace parte de la misma clase con el estímulo muestra. Cada estímulo muestra (A1 y A2) se presentó dos veces de manera semialeatoria. El criterio para continuar con los demás ensayos de entrenamiento fue obtener tres o cuatro aciertos en los cuatro ensayos de prueba. El experimento se terminaba para los participantes que obtuvieran dos aciertos o menos.

El siguiente bloque de ensayos se diferenció del primero en la presentación de un estímulo adicional para cada clase (X1 y X2). Estos estímulos se presentaron $0.3 \mathrm{~cm}$ a la derecha del estímulo muestra (A1 o A2) para formar las muestras compuestas AX1 y AX2. Una vez terminados estos 10 ensayos de entrenamiento se presentó una prueba filtro para evaluar las relaciones AX-B. Solamente los participantes que obtuvieron tres o más aciertos continuaron con la prueba de bloqueo.

En la prueba de bloqueo se presentó X (X1 o X2) como estímulo muestra y B1 y B2 como estímulos de comparación. En esta prueba se evaluó si los 
participantes respondían a la relación X-B dado el entrenamiento previo en A-B y AX-B. La prueba consistió de 10 ensayos en los que los estímulos muestra X1 y X2 se presentaron cinco veces cada una de forma semialeatoria. Se consideró como indicador del efecto bloqueo responder correctamente a menos del $70 \%$ de los ensayos de prueba (X-B). En estos casos el experimento se terminaba con la prueba de bloqueo. Los participantes que respondieron correctamente al 70 \% o más de los ensayos de bloqueo (es decir, no presentaron bloqueo de $\mathrm{X}$-B) continuaban con el entrenamiento de la relación B-Cy posteriormente con las pruebas de relaciones derivadas.

Pruebas de relaciones derivadas: estas pruebas contrastaron la adquisición de relaciones derivadas entre los estímulos A-B-Cy X-B-C. Para todos los grupos se evaluaron las relaciones de simetría B-A, B-X y C-B, las relaciones transitivas A-C y $\mathrm{X}-\mathrm{C}$, las relaciones de equivalencia C-A y C-X y se incluyeron las relaciones simétricas entre los elementos de la muestra compuesta A-X y X-A. Para todas las pruebas se utilizó el arreglo de igualación a la muestra, y cada una de las pruebas incluyó 10 ensayos.

Grupo BL-I. En este procedimiento se invirtió el orden de los entrenamientos A-B y AX-B. Así, las primeras relaciones entrenadas fueron $\mathrm{AX1}$ - $\mathrm{B} 1$ y AX2-B2, seguidas por A1-B1 y A2-B2. Al igual que para el grupo BL, a cada uno de estos bloques de entrenamiento le siguió un bloque de pruebas filtro de cuatro ensayos que, de no cumplirse el criterio, terminaban el experimento. A continuación, se presentó la prueba de bloqueo X-B, seguida del último bloque de entrenamiento B-C para los participantes que obtuvieron más de $7 / 10$ aciertos en la prueba. Una vez terminado el entrenamiento B-C, los participantes continuaron con las pruebas de relaciones derivadas.

Grupo CL. Para este grupo la tarea experimental no incluyó el entrenamiento de las relaciones A-B. Se presentó un bloque de ensayos de entrenamiento AX-B seguido de cuatro ensayos de prueba-filtro AX-B y de la prueba de bloqueo X-B. Al igual que en los procedimientos de los otros dos grupos, los participantes que obtuvieron un porcentaje de

\section{TABLA 1}

Relaciones entrenadas en los grupos BL, BL-I y CL

\begin{tabular}{lll}
\hline BL & BL-I & CL \\
\hline A-B & AX-B & AX-B \\
A-B (prueba filtro) & AX-B (prueba filtro) & AX-B (prueba filtro) \\
AX-B & A-B & \\
AX-B (prueba filtro) & A-B (prueba filtro) & \\
X-B (prueba bloqueo) & X-B (prueba bloqueo) & X-B (prueba bloqueo) \\
B-C & B-C & B-C \\
\hline Pruebas de relaciones derivadas & & \\
\hline B-A s. & B-A s. & B-A s. \\
B-X s. & B-X s. & B-X s. \\
C-B s. & C-B s. & C-B s. \\
A-X s. (Entre elementos de la muestra & A-X s. (Entre elementos de la muestra & A-X s. (Entre elementos de la muestra \\
compuesta) & compuesta) & compuesta) \\
A-C t. & A-C t. & A-C t. \\
X-C t. & X-C t. & X-C t. \\
C-A e. & C-A e. & C-A e. \\
C-X e. & C-X e. & C-X e \\
X-A s. (Entre elementos de la muestra & X-A s. (Entre elementos de la muestra & X-A s. (Entre elementos de la muestra \\
compuesta) & compuesta) & compuesta) \\
\hline
\end{tabular}

Nota. s: Simetría; t: Transitividad; e: Equivalencia.

Fuente: elaboración propia. 
TABLA 2

Respuestas correctas a pruebas de bloqueo y relaciones derivadas para el grupo $\mathrm{BL}$

\begin{tabular}{ccccccccccc}
\hline Ptes & X-B (Bloqueo) & B-A & B-X & C-B & A-X & A-C & X-C & C-A & C-X & X-A \\
\hline 1 & 10 & 0 & 10 & 10 & 0 & 0 & 10 & 0 & 9 & 0 \\
2 & 10 & 9 & 10 & 10 & 10 & 9 & 9 & 10 & 10 & 9 \\
3 & 10 & 0 & 10 & 9 & 2 & 4 & 5 & 2 & 10 & 0 \\
4 & 10 & 10 & 10 & 10 & 10 & 10 & 10 & 10 & 9 & 10 \\
5 & 7 & 5 & 3 & 3 & 2 & 5 & 3 & 9 & 7 & 6 \\
6 & 10 & 10 & 10 & 9 & 10 & 10 & 10 & 10 & 10 & 10 \\
7 & 10 & 1 & 10 & 10 & 3 & 3 & 7 & 1 & 7 & 1 \\
8 & 6 & & & & & & & & & \\
\hline
\end{tabular}

Fuente: elaboración propia.

aciertos superior al $70 \%$ continuaron con el entrenamiento en las relaciones B-C y posteriormente con las pruebas de relaciones derivadas. En la Tabla 1 se presenta un resumen de la secuencia de las fases de entrenamiento y pruebas para cada grupo.

\section{Resultados}

En las tablas que se presentan a continuación se observa el desempeño de cada uno de los participantes en la prueba de bloqueo (X-B) y en cada una de las pruebas de relaciones derivadas. Estas últimas incluyen las pruebas de simetría, transitividad y equivalencia con los estímulos que componen la muestra compuesta AX separadamente, y pruebas de simetría entre los elementos de AX (A-X y X-A). El orden de las columnas corresponde al orden en el que aparecieron las pruebas durante la tarea experimental. La Tabla 2 presenta los resultados obtenidos por los participantes del grupo BL y las
Tablas 3 y 4 a continuación muestran los resultados de los grupos BL-I y CL, respectivamente. Al utilizar el procedimiento tradicional de bloqueo se observó que la historia de exposiciones a A-B no impidió el aprendizaje de B-X durante el entrenamiento posterior con la muestra compuesta AX-B. Como se puede ver en la Tabla 2, para siete de ocho participantes no se observó el fenómeno de bloqueo, y se observa formación de clases de equivalencia en las que se incluye cada uno de los elementos de la muestra compuesta. De estos siete participantes solamente uno obtuvo un desempeño menor al $100 \%$ en la prueba de bloqueo.

Los participantes 6, 4 y 2 obtuvieron desempeños por encima del $90 \%$ para todas las pruebas de relaciones derivadas. El participante 1 obtuvo desempeños perfectos en las pruebas de simetría, transitividad y equivalencia con el estímulo de bloqueo X, pero obtuvo 0 respuestas correctas con el componente A de la muestra compuesta. El parti-

TABLA 3

Respuestas correctas a pruebas de bloqueo y relaciones derivadas para el grupo BL-I

\begin{tabular}{ccccccccccc}
\hline Ptes & X-B (Bloqueo) & B-A & B-X & C-B & A-X & A-C & X-C & C-A & C-X & X-A \\
\hline 1 & 5 & & & & & & & & & \\
2 & 10 & 10 & 10 & 9 & 10 & 10 & 10 & 9 & 9 \\
3 & 5 & & & & & & & & & \\
4 & 6 & & & & & & & & & \\
5 & 6 & 10 & 10 & 10 & 9 & 10 & 10 & 10 & 10 & 10 \\
6 & 10 & 10 & 10 & 10 & 10 & 10 & 10 & 10 & 10 & 10 \\
7 & 10 & 10 & 10 & 10 & 10 & 10 & 10 & 10 & 10 & 10 \\
8 & 9 & & & & & & & & & \\
\hline
\end{tabular}

Fuente: elaboración propia. 
TABLA 4

Respuestas correctas a pruebas de bloqueo y relaciones derivadas para el grupo Control

\begin{tabular}{ccccccccccc}
\hline Ptes & X-B (Bloqueo) & B-A & B-X & C-B & A-X & A-C & X-C & C-A & C-X & X-A \\
\hline 1 & 3 & & & & & & & & & \\
2 & 10 & 2 & 0 & 10 & 0 & 7 & 2 & 2 & 2 & 8 \\
3 & 5 & & & & & & & & & \\
4 & 10 & 9 & 9 & 9 & 10 & 10 & 10 & 10 & 8 & 10 \\
5 & 8 & 4 & 7 & 10 & 3 & 5 & 9 & 5 & 9 & 5 \\
6 & 10 & 10 & 10 & 10 & 10 & 10 & 9 & 10 & 10 & 10 \\
7 & 6 & & & & & & & & & \\
8 & 10 & 10 & 10 & 10 & 10 & 10 & 10 & 10 & 10 & 10 \\
\hline
\end{tabular}

Fuente: elaboración propia.

cipante 5 solamente obtuvo desempeños superiores al $70 \%$ en las pruebas de equivalencia C-A y C-X.

En la Tabla 3 se presenta el número de respuestas correctas a la prueba de bloqueo y a las pruebas de relaciones derivadas para el grupo BL-I. En este grupo solamente la mitad de los participantes respondieron seleccionando el estímulo B1 ante la presentación de X1 y el estímulo B2 ante la presentación de X2; es decir, no se observó bloqueo del aprendizaje de la relación X-B para la mitad de los participantes de este grupo. Los cuatro participantes en los que no se observó el fenómeno de bloqueo obtuvieron un desempeño superior al $90 \%$, tanto en la prueba de bloqueo como en todas las pruebas de relaciones derivadas (sin el estímulo de bloqueo X y con el estímulo X en ausencia de A). Los participantes 7 y 8 obtuvieron un desempeño perfecto en todas las pruebas de relaciones derivadas. El participante 6 obtuvo solamente un error en los ensayos de prueba de la relación A-X y el participante 2 obtuvo desempeños perfectos para cinco de las pruebas y nueve de 10 aciertos en las pruebas restantes.

El procedimiento del grupo control se diferencia de los procedimientos anteriores en que no se presenta un bloqueo de ensayos de entrenamiento A-B adicional al entrenamiento con la muestra compuesta AX-B. En este grupo, tres de ocho participantes no respondieron a la relación X-B. Como se puede ver en la Tabla 4 , de los cinco participantes en los que no se observó bloqueo de la relación $\mathrm{X}-\mathrm{B}$, cuatro obtuvieron desempeños perfectos en la prueba de bloqueo y tres obtuvieron desempe- ños por encima del $80 \%$ en todas las pruebas de relaciones derivadas.

La inspección visual de los datos presentados en las Tablas 2, 3 y 4 muestra que para los participantes expuestos al procedimiento de bloqueo tradicional no se observó el efecto de bloqueo, mientras que en los grupos de BL-I y el grupo Control se observó bloqueo solamente en algunos casos.

En el grupo BL-I se observa el mayor número de participantes en los que se observó bloqueo de la respuesta a la relación X-B (4 de 8). Dado un entrenamiento con una muestra compuesta AX-B y un entrenamiento adicional $A-B$ posterior, no se adquirieron respuestas con respecto a la relación X-B. Sin embargo, la otra mitad de los participantes obtuvo un alto desempeño en la prueba de la relación X-B y en las pruebas de relaciones derivadas con los estímulos X y A presentados separadamente. Adicionalmente, no se aprecia una diferencia importante en el número de participantes en los que se observó bloqueo, en el grupo BL-I (4/4) y el grupo Control (3/4). Contrasta con los resultados de estos dos grupos que en el grupo BL solamente un participante no alcanzó el criterio de aprendizaje de la relación X-B (1/8).

Con el fin de determinar si estos hallazgos reflejan diferencias entre las tres condiciones de entrenamiento, se presentan las características de distribución de los datos obtenidos en la prueba de bloqueo para cada uno de los grupos. La Tabla 5, muestra que mientras que las medias observadas para los grupos BL-I y Control son similares, la media del grupo BL es más alta que la de ambos grupos. 


\section{TABLA 5}

Estadísticos descriptivos para el número de respuestas correctas a la prueba de bloqueo X-B en cada grupo

\begin{tabular}{ccc}
\hline Control & Media & 7.7 \\
& IC 95\% -inferior & 5.4 \\
& IC 95\% -superior & 10 \\
& Mediana & 9 \\
& Mínimo & 3 \\
& Máximo & 10 \\
BL & Rango & 7 \\
\hline BL-I & Media & 9.1 \\
& IC 95\% -inferior & 7.7 \\
& IC 95\% -superior & 10 \\
Mediana & 10 \\
& Mínimo & 6 \\
& Máximo & 10 \\
Rango & 4 \\
\hline IC 95\% -inferior & 7.6 \\
& IC 95\% -superior & 5.6 \\
Mediana & 9.5 \\
Mínimo & 5 \\
& Máximo & 10 \\
Rango & 5 \\
\hline
\end{tabular}

Fuente: elaboración propia.

Sin embargo, la superposición de los intervalos de confianza de los tres grupos sugiere que las distribuciones son similares.

Debido a esto, y con el fin de contrastar la igualdad de los parámetros de centralización de las distribuciones, se utilizó la prueba KruskalWallis para muestras independientes (SPSS 18). Al obtener $p=0.27$, se rechaza la hipótesis de que las medianas de cada grupo son significativamente diferentes. Esto indica que las distribuciones de los datos de desempeño en la prueba X-B son similares para los tres grupos.

\section{Discusión}

El propósito de este estudio fue examinar si la redundancia de $\mathrm{X}$ en la predicción del EI constituye un factor crítico en la explicación del fenómeno de bloqueo cuando este se presenta en la formación de clases de equivalencia. En particular, se examinó si los procedimientos de bloqueo y bloqueo invertido tienen un impacto diferencial en la transferencia de funciones entre miembros de las clases. Si bien los procedimientos pavlovianos se han usado ampliamente para dar cuenta de fenómenos cognitivos en humanos (p. ej., Didden et al., 2000; Livesey \& Boakes, 2004; Melchers et al., 2008; Pineño et al., 2006), pocos estudios han utilizado este paradigma teórico y procedimental en el estudio de la formación de clases de estímulos.

En oposición a los postulados de Sidman (1992, 2000), algunos estudios han mostrado sistemáticamente que las contingencias operantes no constituyen una condición necesaria para la formación de clases derivadas y que los procedimientos pavlovianos producen transferencia de funciones y emergencia de relaciones de equivalencia entre estímulos arbitrarios de manera suficiente (Delgado \& Medina, 2011; Delgado et al., 2011; Leader \& Barnes-Holmes, 2001; Tonneau \& González, 2004). Dado lo anterior, el efecto bloqueo podría explicar cuáles son las circunstancias en las que se limita o se impide la transferencia de funciones entre miembros de una clase de estímulos.

En este estudio, se examinó si el entrenamiento adicional entre los miembros de una clase (por ejemplo, A1-B1) impide la inclusión de un tercer miembro (X1) cuando este se presenta simultáneamente con A1 para formar una muestra compuesta (A1X1). Los resultados muestran que el efecto bloqueo no se presentó de manera consistente en todos los casos como sucede en los estudios con animales no humanos.

La comparación entre los grupos BL, BL-I y CL sugiere que la historia adicional, bien sea previa o posterior, de asociaciones $\mathrm{A}-\mathrm{B}$ a asociaciones $\mathrm{AX}-\mathrm{B}$ no tiene ningún efecto en el bloqueo de la transferencia de funciones entre miembros de una clase de estímulos arbitrarios. Si la hipótesis clásica de redundancia del estímulo $\mathrm{X}$ en la predicción de $\mathrm{B}$ (i. e., el EI) se demostrara para el caso de los humanos, se habría observado el efecto bloqueo para la mayoría de los participantes de los grupos de bloqueo (BL y BL-I), pero no para el grupo control.

En contraste, se observó el efecto bloqueo para la mitad de los participantes del grupo control y 
del grupo BL-I. Si bien las diferencias entre los tres grupos no fueron estadísticamente significativas, la inspección visual de los resultados muestra que no se presentó el efecto bloqueo para la mayoría de participantes del grupo BL (7 de 8). Posiblemente sea más fácil incluir un nuevo elemento a la clase A-B cuando se presenta $X$ en la fase de entrenamiento $\mathrm{AX}-\mathrm{B}$, que aprender la relación entre tres eventos y después de presentaciones adicionales de solo dos de ellos responder en términos del elemento faltante. El primer caso corresponde al procedimiento tradicional de bloqueo, mientras que el segundo corresponde al procedimiento de bloqueo invertido.

Se sugiere replicar el estudio con un mayor número de participantes por grupo para establecer si existen efectos diferenciales entre los procedimientos de bloqueo, bloqueo invertido y control, sobre el bloqueo en la formación de relaciones derivadas. Adicionalmente, en estudios similares se podrían utilizar más de dos clases de equivalencia; de esta manera, en cada ensayo habría tres estímulos de comparación y no únicamente dos. Al utilizar solamente dos estímulos de comparación para cada ensayo, es posible que se responda con respecto a una sola clase, y que las respuestas a la otra sean dadas por exclusión.

Resulta notable observar que no solo los procedimientos tradicionales de bloqueo no impidieron que se respondiera a $\mathrm{X}$ como a un miembro adicional de la clase para algunos participantes, sino que en las pruebas de relaciones derivadas se observó un desempeño superior al $80 \%$ para los tres grupos en general. Muchos de estos participantes obtuvieron desempeños del $90 \%$ y del $100 \%$ aún en las pruebas de simetría, transitividad y equivalencia que incluyeron el estímulo de bloqueo $(\mathrm{X})$ en ausencia del otro miembro (A) de la muestra compuesta (AX).

La ausencia del efecto bloqueo en la mayoría de los casos sugiere que el factor crítico en la presentación de este fenómeno no es la redundancia del estímulo X o la exposición adicional a ensayos con un solo elemento del compuesto, sino la percepción de este estímulo como un todo o como estímulos separados. Se puede pensar entonces que no se observó bloqueo en los participantes que respondieron a los estímulos de forma elemental, es decir, atendiendo a cada uno de los elementos de forma separada, y que fue precisamente este tipo de percepción lo que les permitió responder a A y a X como miembros de una clase de equivalencia. Por otra parte, es posible que los casos en los que se observa bloqueo correspondan con formas de responder de tipo configural. Al percibir a AX como un solo evento, no hay discriminación de sus elementos y, por lo tanto, no se responde a X solo como a un miembro de la clase.

A este respecto, resulta interesante observar el desempeño del participante 1 quien obtuvo desempeños perfectos en las pruebas de simetría, transitividad y equivalencia con el estímulo de bloqueo (X) y cero respuestas correctas en las mismas pruebas con el componente A de la muestra compuesta. Esto sugiere que este participante respondió separadamente a cada elemento de la muestra compuesta AX.

Sin embargo, algunos estudios aportan evidencia que sugiere lo contrario; es decir, que las percepciones elementales son predictivas del efecto bloqueo mientras que las configurales no lo son (De Houwer et al., 2002; Livesey \& Boakes, 2004). La explicación que proponen los autores es que al responder a $\mathrm{A}$ y a $\mathrm{X}$ de forma elemental, la historia previa de exposición a A-EI tiene un efecto sobre el posterior entrenamiento AX-EI. De esta manera, en este segundo entrenamiento, el EI se predice completamente por el estímulo A, y por lo tanto X se hace redundante. Si bien esta interpretación es consistente con la hipótesis de la redundancia de $\mathrm{X}$ en la predicción del EI, en el presente estudio se propone una interpretación alterna con base en los resultados obtenidos.

Dadas las inconsistencias en los hallazgos y la falta de consenso en las interpretaciones de los mismos, se considera necesario aportar evidencia empírica adicional acerca de los efectos del responder configural o elementalmente, sobre el efecto bloqueo en la formación de clases. Particularmente, sería interesante manipular la presentación del estímulo compuesto de manera tal que se enfaticen percepciones configurales y elementales de los estímulos compuestos. Para esto, habría que considerar cuáles son las características relacionales o morfoló- 
gicas de los eventos que predicen la probabilidad de uno u otro tipo de respuestas (ver, Glautier, 2002; Melchers et al., 2008).

Además del estudio de las condiciones que impiden o dificultan el aprendizaje, futuras investigaciones podrían examinar si se observa el efecto contrario o de contrabloqueo en la adquisición de clases de estímulos. En este fenómeno se observa que en lugar de obstaculizar el condicionamiento del EC2, el estimulo previamente condicionado facilita el condicionamiento de este (Batsell \& Batson, 1999; Batson \& Batsell, 2000).

Si bien el procedimiento de igualación a la muestra es el más utilizado para el estudio de formación de clases de equivalencia, no es el único procedimiento ni el que más facilita la observación del fenómeno cuando se estudia en diversos grupos poblacionales o en animales no humanos. Más aún, una de sus dificultades consiste en que contribuye a que el procedimiento mismo se confunda con el fenómeno (Hayes, 1992; Rehfeldt \& Hayes, 1998; Tonneau \& Sokolowsky, 1997; Vaughan, 1989). Futuros desarrollos podrían explorar las características de los estímulos compuestos que facilitan o impiden la formación de clases, utilizando arreglos experimentales más sencillos y con mayor validez ecológica como los que se describen en la literatura pavloviana sobre cognición humana.

\section{Referencias}

Arcediano, F., Matute, H. \& Miller, R. R. (1997). Blocking of Pavlovian conditioning in humans. Learning and Motivation, 28(2), 188-199.

Augustson, E. M., Dougher, M. J. \& Markham, M. R. (2000). Emergence of conditional stimulus relations and transfer of respondent eliciting functions among compound stimuli. The Psychological Record, 50(4), 745-770.

Batsell, W. R., Jr. \& Batson, J. D. (1999). Augmentation of taste conditioning by a preconditioned odor. Journal of Experimental Psychology: Animal Behavior Processes, 25(3), 374-388.

Batson, J. D. \& Batsell, W. R., Jr. (2000). Augmentation, not blocking, in an $\mathrm{A}+/ \mathrm{AX}+$ flavor-conditioning procedure. Psychonomic Bulletin $\mathcal{E}$ Review, 7(3), 466-471.

Blaisdell, A. P., Gunther, L. G. \& Miller, R. R. (1999). Recovery from blocking achieved by extinguishing the blocking CS. Animal Learning 83 Behavior, 27(1), 63-76.

Carpentier, F., Smeets, P. M. \& Barnes-Holmes, D. (2005). Igualación de muestras compuestas con comparaciones sencillas: la intercambiabilidad de los términos estimulares. Revista Latinoamericana de Psicología, 37(2), 317-331.

Cepeda-Islas, M. L., López-Hernández, A., MorenoRodríguez, D., Plancarte-Cancino, P., ArroyoHernández, R. M. \& Hickman-Rodríguez, H. (2009). Efecto de las descripciones y el reforzamiento sobre la generación de reglas. Universitas Psychologica, 8(2), 371-384.

Crookes, A. E. \& Moran, P. M. (2003). An investigation into age and gender differences in human Kamin blocking using a computerised task. Developmental Neuropsychology, 24(1), 39-55.

Davey, G. C. L. \& Arulampalam, T. (1982). Second order fear conditioning in humans: Persistence of CR2 following extinction of CR1. Behavioral Research and Therapy, 20(4), 391-396.

De Houwer, J., Beckers, T. \& Glautier, S. (2002). Outcome and cue properties modulate blocking. Quarterly Journal of Experimental Psychology-Section A, 55(3), 965-985.

Delgado, D. \& Medina, I. (2011). Efectos de dos tipos de entrenamiento respondiente sobre la formación de relaciones de equivalencia. Revista Mexicana de Análisis de la Conducta, 37(1), 33-50. Disponible en http://www.revistas.unam.mx/index.php/rmac/ article/view/19475

Delgado, D., Medina, I. \& Soto, S. (2011). El lenguaje como mediador de la transferencia de funciones: ies necesario nominar para relacionar? Revista Mexicana de Análisis de la Conducta, 37(2), 31-52. Disponible en http://dx.doi.org/10.5514/rmac.v37. i2. 26138

Denniston, J. C., Miller, R. R. \& Matute, H. (1996). Biological significance as a determinant of cue competition. Psychological Science, 7(6), 325-331.

Didden, R., Prinsen, H. \& Sigafoos, J. (2000).The blocking effect of pictorial prompts on sight-word read- 
ing. Journal of Applied Behavior Analysis, 33(3), 317-320.

Dougher, M. J. \& Markham, M. R. (1994). Stimulus equivalence, functional equivalence and the transfer of function. En S. C. Hayes, L. J. Hayes, M. Sato $\&$ K. Ono (Eds.), Behavior analysis of language and cognition (pp. 71-90). Reno, NV: Context Press.

Escuer-Acín, E., García-García, A., Bohórquez-Zayas, C. \& Gutiérrez-Domínguez, M. T. (2006). Formación de clases de equivalencia aplicada al aprendizaje de notas musicales. Piscothema, 18(1), 31-36.

García, A. \& Benjumea, S. (2007). Facilitación de relaciones bidireccionales en palomas usando una tarea de transferencia tras entrenamiento en discriminación de la propia conducta. Universitas Psychologica, 6(2), 441-450.

Glautier, S. (2002). Separation of target and competitor cues enhances human blocking in human causality judgments. The Quarterly Journal of Experimental Psychology-Section B, 55(2), 121-135.

Hernández, A., Céspedes, S. \& Prieto, L. (2007). Relaciones de equivalencia con estímulos compuestos. Suma Psicológica, 14(1), 51-72.

Hayes, L. J. (1992). Equivalence as a process. En S. C. Hayes \& L. J. Hayes (Eds.), Understanding verbal relations (pp. 97-108). Reno, NV: Context Press.

Kamin, L. J. (1968). "Attention-like" processes in classical conditioning. En M. R. Jones (Ed.), Miami Symposium on the Prediction of Behavior, 1967: Aversive stimulation (pp. 9-31). Coral Gables, Fl: University of Miami Press.

Kamin, L. J. (1969). Predictability, surprise, attention and conditioning. En B. A. Campbell \& R. M. Church (Eds.), Punishment and aversive behavior (pp. 279-296). New York: Appleton-CenturyCrofts.

Kruschke, J. K., Kapperman, E. S. \& Hetrick, W. P. (2005). Eye gaze and individual differences consistent with learned attention and associative blocking and highlighting. Journal of Experimental Psychology: Learning, Memory and Cognition, 31(5), 830-845.

Laane, K., Aru, J. \& Dickinson, A. (2010). Non-competitive liking for brands. No blocking in evaluative conditioning. Appetite, 54(1), 100-107.
Lachnit, H. \& Lober, K. (2001). What is learned in patterning discriminations? Further tests of configural accounts of associative learning in human electrodermal conditioning. Biological Psychology, 56(1), 45-61.

Leader, G. \& Barnes-Holmes, D. (2001). Matching-tosample and respondent-type training as methods for producing equivalence relations: Isolating the critical variable. The Psychological Record, 51(3), 429-444.

Livesey, E. J. \& Boakes, R. A. (2004). Outcome additivity, elemental processing and blocking in human causality judgments. The Quarterly Journal of Experimental Psychology-Section B, 57(4), 361-379.

Mackintosh, N. J. (1975). A theory of attention: Variations in the associability of stimuli with reinforcement. Psychological Review, 82(4), 276-298.

Markham, M. R. \& Dougher, M. J. (1993). Compound stimuli in emergent stimulus relations: Extending the scope of stimulus equivalence. Journal of the Experimental Analysis of Behavior, 60(3), 529-542.

Markham, M. R. \& Dougher, M. J. \& Augustson, E. M. (2002). Transfer of operant discrimination and respondent elicitation via emergent relations of compound stimuli. The Psychological Record, 52(3), 325-350.

McLaren, I. P. L. \& Mackintosh, N. J. (2000). An elemental model of associative learning: I. Latent inhibition and perceptual learning. Animal Learning E⿱ Behavior, 28(3), 211-246.

Melchers, K. G., Shanks, D. R. \& Lachnit, H. (2008). Stimulus coding in human associative learning. Flexible representations of parts and wholes. Behavioural Processes, 77(3), 413-427.

Pineño, O., Urushihara, K, Stout, S., Fuss, J. \& Miller, R. R. (2006). When less is more: Extending training of the blocking association following compound training attenuates the blocking effect. Learning EB Behavior, 34(1), 21-36.

Rehfeldt, R. A., Dixon, M. R., Hayes, L. J. \& Steele, A. (1998). Stimulus equivalence and the blocking effect. The Psychological Record, 48(4), 647-664.

Rehfeldt, R. A. \& Hayes, L. J. (1998). The operantrespondent distinction revisited: Toward an understanding of stimulus equivalence. The Psychological Record, 48(2), 187-210. 
Rescorla, R. A. (1988). Pavlovian conditioning is not what you think it is. American Psychologist, 43(3), 151-160.

Rescorla, R. A. (1999). Learning about qualitatively different outcomes during a blocking procedure. Animal Learning $\mathcal{E}$ Behavior, 27(2), 140-151.

Shanks, D. R. (1985). Forward and backward blocking in human contingency judgment. Quarterly Journal of Experimental Psychology-Section B, 37(1), 1-21.

Sidman, M. (1992). Equivalence relations: Some basic considerations. En S. C. Hayes \& L. J. Hayes (Eds.), Understanding verbal relations (pp. 15-27). Reno, NV: Context Press.

Sidman, M. (2000). Equivalence relations and the reinforcement contingency. Journal of the Experimental Analysis of Behavior, 74(1), 127-146.

Sidman, M. \& Tailby, W. (1982). Conditional discrimination vs. matching to sample: An expansion of the testing paradigm. Journal of the Experimental Analysis of Behavior, 37(1), 5-22.

Singh, N. \& Solman, R. (1990). A stimulus control analysis of the picture-word problem in children who are mentally retarded: The blocking effect. Journal of Applied Behavior Analysis, 23(4), 525-532.

Stout, S. C. \& Miller, R. R. (2007). Sometimes competing retrieval (SOCR): A formalization of the comparator hypothesis. Psychological Review, 114(3), 759-783.

Stromer, R. \& Stromer, J. B. (1990). The formation of arbitrary stimulus classes in matching to complex samples. The Psychological Record, 40(1), 51-66.

Tonneau, F., Arreola, F. \& Martínez, A. (2006). Function transformation without reinforcement. Journal of Experimental Analysis of Behavior, 85(3),
393-405. Disponible en http://dx.doi.org/10.1901/ jeab.2006.49-05

Tonneau, F. \& González, C. (2004). Function transfer in human operant experiments: The role of stimulus pairings. Journal of Experimental Analysis of Behavior, 81(3), 239-255. Disponible en http:// dx.doi.org/10.1901/jeab.2004.81-239

Tonneau, F. \& Sokolowsky, M. B. C. (1997). Standard principles, nonstandard data and unsolved issues. Journal of the Experimental Analysis of Behavior, 68(2), 266-270.

Valero-Aguayo, L. \& Luciano-Soriano, C. (1992). Relaciones de equivalencia: una síntesis teórica y los datos empíricos a nivel básico y aplicado. Psicothema, 4(2), 413-428.

Vaughan, W. (1989). Reply to Hayes. Journal of Experimental Analysis Behavior, 51(3), 397-397.

Vives-Montero, C., Valero-Aguayo, L. \& Ascanio, L. (2011). Generalización en el manejo del dinero mediante un entrenamiento en relaciones de equivalencia. Psicothema, 23(1), 31-37.

Wasserman, E. A. \& Berglan, L. R. (1998). Backward blocking and recovery from overshadowing in human causal judgment: The role of within-compound associations. Quarterly Journal of Experimental Psychology-Section B, 51(2), 121-138.

Wilson, P. N. \& Alexander, T. (2008). Blocking of spatial learning between enclosure geometry and a local landmark. Journal of Experimental Psychology: Learning, Memory E Cognition, 34(6), 1369-1376.

Wulfert, E. \& Hayes, S. C. (1988). Transfer of a conditional ordering response through conditional equivalence classes. Journal of the Experimental Analysis of Behavior, 50(2), 125-144. 